\title{
Recognizing HH-free, HHD-free, and Welsh-Powell Opposition Graphs
}

\author{
Stavros D. Nikolopoulos and Leonidas Palios \\ Department of Computer Science, University of Ioannina, P.O.Box 1186, GR-45110 Ioannina, Greece \\ \{stavros, palios\}@es.uoi.gr
}

received Oct 7, 2004, revised Jun 27, 2005, Nov 11, 2005, accepted Jan 8, 2006.

\begin{abstract}
In this paper, we consider the recognition problem on three classes of perfect graphs, namely, the HH-free, the HHDfree, and the Welsh-Powell opposition graphs (or WPO-graphs). In particular, we prove properties of the chordal completion of a graph and show that a modified version of the classic linear-time algorithm for testing for a perfect elimination ordering can be efficiently used to determine in $O(n \min \{m \alpha(n, n), m+n \log n\})$ time whether a given graph $G$ on $n$ vertices and $m$ edges contains a house or a hole; this implies an $O(n \min \{m \alpha(n, n), m+$ $n \log n\})$-time and $O(n+m)$-space algorithm for recognizing HH-free graphs, and in turn leads to an HHD-free graph recognition algorithm exhibiting the same time and space complexity. We also show that determining whether the complement $\bar{G}$ of the graph $G$ is HH-free can be efficiently resolved in $O(n m)$ time using $O\left(n^{2}\right)$ space, which leads to an $O(n \mathrm{~m})$-time and $O\left(n^{2}\right)$-space algorithm for recognizing WPO-graphs. The previously best algorithms for recognizing HH-free, HHD-free, and WPO-graphs required $O\left(n^{3}\right)$ time and $O\left(n^{2}\right)$ space.
\end{abstract}

Keywords: HH-free graph, HHD-free graph, Welsh-Powell opposition graph, perfectly orderable graph, recognition.

\section{Introduction}

A linear order $\prec$ on the vertices of a graph $G$ is perfect if the ordered graph $(G, \prec)$ contains no induced $P_{4}$ abcd with $a \prec b$ and $d \prec c$ (such a $P_{4}$ is called an obstruction). In the early 1980s, Chvátal [2] defined the class of graphs that admit a perfect order and called them perfectly orderable graphs. The interest in perfectly orderable graphs comes from the fact that several problems in graph theory, which are NP-complete in general graphs, have polynomial-time solutions in graphs that admit a perfect order [1, 5]; unfortunately, it is NP-complete to decide whether a graph admits a perfect order [12]. Since the recognition of perfectly orderable graphs is NP-complete, we are interested in characterizing graphs which form polynomially recognizable subclasses of perfectly orderable graphs. Many such classes of graphs, with very interesting structural and algorithmic properties, have been defined so far and shown to admit polynomial-time recognitions (see [1; 5]); note however that not all subclasses of perfectly orderable graphs admit polynomial-time recognition [7].

In this paper, we consider the class of HH-free graphs and two classes of perfectly orderable graphs, namely, the HHD-free, and the Welsh-Powell opposition graphs; note that the complement of an HH-free graph is perfectly orderable (this was conjectured by Chvátal and proved by Hayward [6]). A graph is 


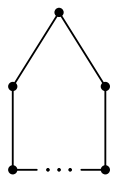

hole

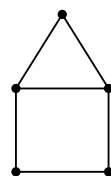

house

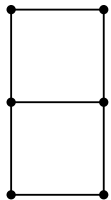

domino

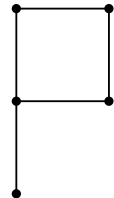

$\mathrm{P}$

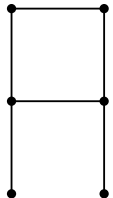

A

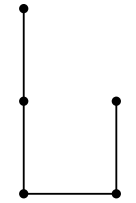

$P_{5}$

Fig. 1: Some simple graphs.

$H H$-free if it contains no hole (i.e., a chordless cycle on $\geq 5$ vertices) and no house as induced subgraphs (see Figure 1). The HH-free graphs properly generalize the class of triangulated (or chordal) graphs, i.e., graphs with no induced chordless cycles of length greater than or equal to four [5]. A subclass of HH-free graphs, which also properly generalizes the class of triangulated graphs, is the class of HHD-free graphs; a graph is $H H D$-free if it contains no hole, no house, and no domino as induced subgraphs (see Figure 17. In [8], Hoàng and Khouzam proved that the HHD-free graphs admit a perfect order, and thus are perfectly orderable.

A graph is called an Opposition graph if it admits a linear order $\prec$ on its vertices such that there is no $P_{4} a b c d$ with $a \prec b$ and $c \prec d$. Opposition graphs belong to the class of bip* graphs (see [1]), and hence are perfect [13]. The complexity of recognizing opposition graphs is unknown. It is also open whether there is an opposition graph that is not perfectly orderable [1]. The class of opposition graphs contains several known classes of perfectly orderable graphs. For example, bipolarizable graphs are, by definition, opposition graphs; a graph is bipolarizable if it admits a linear order $\prec$ on its vertices such that every $P_{4} a b c d$ has $b \prec a$ and $c \prec d$ [14]. Another subclass of opposition graphs, which we study in this paper, are the Welsh-Powell opposition graphs. A graph is defined to be a Welsh-Powell Opposition graph (or WPO-graph for short), if it is an opposition graph for every Welsh-Powell ordering; a Welsh-Powell ordering for a graph is an ordering of its vertices in nondecreasing degree [18].

Hoàng and Khouzam [8], while studying the class of brittle graphs (a well-known class of perfectly orderable graphs which contains the HHD-free graphs), showed that HHD-free graphs can be recognized in $O\left(n^{4}\right)$ time, where $n$ denotes the number of vertices of the input graph. An improved result was obtained by Hoàng and Sritharan [9] who presented an $O\left(n^{3}\right)$-time algorithm for recognizing HH-free graphs and showed that HHD-free graphs can be recognized in $O\left(n^{3}\right)$ time as well; one of the key ingredients in their algorithms is the reduction to the recognition of triangulated graphs. Recently, Eschen et al. [4] described recognition algorithms for several classes of perfectly orderable graphs, among which a recognition algorithm for HHP-free graphs; a graph is HHP-free if it contains no hole, no house, and no "P" as induced subgraphs (see Figure 1). Their algorithm is based on the property that every HHP-free graph is HHDA-free graph (a graph with no induced hole, house, domino, or "A"), and thus a graph $G$ is HHP-free graph if and only if $G$ is a HHDA-free and contains no "P" as an induced subgraph. The characterization of HHDA-free graphs due to Olariu (a graph $G$ is HHDA-free if and only if every induced subgraph of $G$ either is triangulated or contains a non-trivial module [14]) and the use of modular decomposition [11] allowed Eschen et al. to present an $O(n \mathrm{~m})$-time recognition algorithm for HHP-free graphs.

For the class of WPO-graphs, Olariu and Randall [15] gave the following characterization: a graph $G$ is WPO-graph if and only if $G$ contains no induced $C_{5}$ (i.e., a hole on 5 vertices), house, $P_{5}$, or "P" (see Figure 1). It follows that $G$ is a WPO-graph if and only if $G$ is HHP-free and $\bar{G}$ is HH-free. Eschen et al. [4] combined their $O(n m)$-time recognition algorithm for HHP-free graphs with the $O\left(n^{3}\right)$-time recognition 
algorithm for HH-free graphs proposed in [9], and showed that WPO-graphs can be recognized in $O\left(n^{3}\right)$ time.

In this paper, we present efficient algorithms for recognizing HH-free, HHD-free, and WPO-graphs. We show that a variant of the classic linear-time algorithm for testing whether an ordering of the vertices of a graph is a perfect elimination ordering can be used to determine whether a vertex of a graph $G$ belongs to a hole or is the top of a house or a building in $G$. We take advantage of properties characterizing the chordal completion of a graph and show how to efficiently compute for each vertex $v$ the leftmost among $v$ 's neighbors in the chordal completion which are to the right of $v$, without explicitly computing the chordal completion. As a result, we obtain an $O(n \min \{m \alpha(n, n), m+n \log n\})$-time and $O(n+m)$-space algorithm for determining whether a graph on $n$ vertices and $m$ edges is HH-free; we also describe how the algorithm can be augmented to provide a certificate (an induced house or hole) whenever it decides that the input graph is not $\mathrm{HH}$-free.

Our HH-free graph recognition algorithm, in conjunction with results by Jamison and Olariu [10] and by Hoàng and Khouzam [8], enables us to describe an algorithm for recognizing HHD-free graphs which also runs in $O(n \min \{m \alpha(n, n), m+n \log n\})$ time and requires $O(n+m)$ space. Additionally, for a graph $G$ on $n$ vertices and $m$ edges, we show that we can detect whether the complement $\bar{G}$ of $G$ is HH-free in $O(n m)$ time using $O\left(n^{2}\right)$ space. In light of the characterization of WPO-graphs due to Olariu and Randall [15] which implies that a graph $G$ is a WPO-graph if and only if $G$ is HHP-free and its complement $\bar{G}$ is HH-free, and the $O(n m)$-time recognition algorithm for HHP-free graphs of Eschen $e t$ al. [4], our result yields an $O(n m)$-time and $O\left(n^{2}\right)$-space algorithm for recognizing WPO-graphs.

The paper is structured as follows. In Section 2, we review the terminology that we use throughout the paper and we present well known results that are useful for our algorithms. In Section 3, we present the methodology and establish properties that enable us to efficiently determine whether a given graph is HH-free, describe the algorithm, and give its analysis and the certificate computation. The recognition algorithms for HHD-free graphs and WPO-graphs are described and analyzed in Section 4 Section 5 summarizes our results and presents some open problems.

\section{Preliminaries}

We consider finite undirected graphs with no loops or multiple edges. Let $G$ be such a graph; then, $V(G)$ and $E(G)$ denote the set of vertices and of edges of $G$ respectively. The subgraph of a graph $G$ induced by a subset $S$ of $G$ 's vertices is denoted by $G[S]$. A subset $B \subseteq V(G)$ of vertices is a module if $2 \leq|B|<|V(G)|$ and each vertex $x \in V(G)-B$ is adjacent to either all vertices or no vertex in $B$. The neighborhood $N(x)$ of a vertex $x \in V(G)$ is the set of all the vertices of $G$ which are adjacent to $x$. The closed neighborhood of $x$ is defined as $N[x]:=N(x) \cup\{x\}$. We use $M(x)$ to denote the set $V(G)-N[x]$ of non-neighbors of $x$. Furthermore, for a vertex $y \in M(x)$, we use $n(x, y)$ to denote the number of vertices in the set $N(x) \cap N(y)$, i.e., the set of common neighbors of $x$ and $y$, or equivalently, the degree of the vertex $y$ in the graph induced by the set $N(x) \cup\{y\}$. The degree of a vertex $x$ in a graph $G$, denoted $\operatorname{deg}(x)$, is the number of edges incident on $x$; thus, $\operatorname{deg}(x)=|N(x)|$.

A path $v_{0} v_{1} \cdots v_{k}$ of a graph $G$ is called simple if none of its vertices occurs more than once; it is called a cycle (simple cycle) if $v_{0} v_{k} \in E(G)$. A simple path (cycle) is chordless if $v_{i} v_{j} \notin E(G)$ for any two non-consecutive vertices $v_{i}, v_{j}$ in the path (cycle). A chordless path (chordless cycle, respectively) on $n$ vertices is commonly denoted by $P_{n}\left(C_{n}\right.$, respectively). In particular, a chordless path on 4 vertices is denoted by $P_{4}$. If $a b c d$ is a $P_{4}$ of a graph, then the vertices $b$ and $c$ are called midpoints and the vertices $a$ 
Algorithm $\operatorname{PEO}(G, \sigma)$

1. for each vertex $u \in V(G)$ do

$$
A(u) \leftarrow \emptyset ;
$$

2. for $i \leftarrow 1$ to $n-1$ do

3. $u \leftarrow \sigma(i)$;

4. $\quad X \leftarrow\left\{x \in N(u) \mid \sigma^{-1}(u)<\sigma^{-1}(x)\right\}$;

$\{$ neighbors to the right of $u$ (w.r.t. $\sigma)\}$

5. if $X \neq \emptyset$

6. $\quad$ then $w \leftarrow \sigma\left(\min \left\{\sigma^{-1}(x) \mid x \in X\right\}\right)$;

$\{$ leftmost (w.r.t. $\sigma$ ) among vertices in $X\}$

7. concatenate $X-\{w\}$ to $A(w)$;

8. $\quad$ if $A(u)-N(u) \neq \emptyset$ then return("false");

9. return("true");

Fig. 2: The perfect elimination ordering testing algorithm.

and dendpoints of the $P_{4}$ abcd.

Let $G$ be a graph and let $x, y$ be a pair of vertices. If $G$ contains a path from vertex $x$ to vertex $y$, we say that $x$ is connected to $y$. The graph $G$ is connected if $x$ is connected to $y$ for every pair of vertices $x, y \in V(G)$. The connected components (or components) of $G$ are the equivalence classes of the "is connected to" relation on the vertex set $V(G)$. The co-connected components (or co-components) of $G$ are the connected components of the complement $\bar{G}$ of the graph $G$.

A graph $G$ has a perfect elimination ordering if its vertices can be linearly ordered $\left(v_{1}, v_{2}, \ldots, v_{n}\right)$ so that each vertex $v_{i}$ is simplicial in the graph $G_{i}=G\left[\left\{v_{i}, v_{i+1}, \ldots, v_{n}\right\}\right]$ induced by the vertices $v_{i}, v_{i+1}, \ldots, v_{n}$, for $1 \leq i \leq n$; a vertex of a graph is simplicial if its neighborhood induces a complete subgraph. It is well-known that a graph is triangulated if and only if it has a perfect elimination ordering [1, 5; 16]. The notion of a simplicial vertex was generalized by Jamison and Olariu [10] who defined the notion of a semi-simplicial vertex: a vertex of a graph $G$ is semi-simplicial if it is not a midpoint of any $P_{4}$ of $G$. A graph $G$ has a semi-perfect elimination ordering if its vertices can be linearly ordered $\left(v_{1}, v_{2}, \ldots, v_{n}\right)$ so that each vertex $v_{i}$ is semi-simplicial in the graph $G_{i}$, for $1 \leq i \leq n$. A graph is a semi-simplicial graph if and only if it has a semi-perfect elimination ordering (see [4]).

Let $\sigma=\left(v_{1}, v_{2}, \ldots, v_{n}\right)$ be an ordering of the vertices of a graph $G ; \sigma(i)$ is the $i$-th vertex in $\sigma$, i.e., $\sigma(i)=v_{i}$, while $\sigma^{-1}\left(v_{i}\right)$ denotes the position of vertex $v_{i}$ in $\sigma$, i.e., $\sigma^{-1}\left(v_{i}\right)=i, 1 \leq i \leq n$. In Figure 2 , we include the classic algorithm $\operatorname{PEO}(G, \sigma)$ for testing whether the ordering $\sigma$ is a perfect elimination ordering; if the graph $G$ has $n$ vertices and $m$ edges, the algorithm runs in $O(n+m)$ time and requires $O(n+m)$ space [5; 16]. Note that, in $\operatorname{Step} 4$ of the Algorithm $\operatorname{PEO}(G, \sigma)$, the set $X$ is assigned the neighbors of the vertex $u$ which have larger $\sigma^{-1}$ ( )-values; that is, $X=N(u) \cap\{\sigma(i+1), \ldots, \sigma(n)\}$; thus, in Step 6 the vertex $w$ is the neighbor of $u$ in $G$ which is first met among the vertices to the right of $u$ along the ordering $\sigma$. 


\section{Recognizing $\mathrm{HH}$-free graphs}

The most important ingredient (and the bottleneck too) of the HHD-free graph recognition algorithm of Hoàng and Sritharan [9] is an algorithm to determine whether a simplicial vertex $v$ of a graph $G$ is high, i.e., it is the top of a house or a building ${ }^{(i)}$ (or belongs to a hole) in $G$, which involves the following steps:

$\triangleright$ They compute an ordering of the set $M(v)$ of non-neighbors of $v$ in $G$ where, for two vertices $y, y^{\prime} \in M(v), y$ precedes $y^{\prime}$ whenever $n(v, y) \leq n\left(v, y^{\prime}\right)$; recall that, for $y \in M(v), n(v, y)$ is the number of common neighbors of $v$ and $y$. As we will be using this ordering in the description of our approach, we call it a DegMN-ordering of $M(v)$.

$\triangleright$ They perform chordal completion on $G[M(v)]$ with respect to a DegMN-ordering of $M(v)$.

$\triangleright$ The vertex $v$ is high if and only if the graph $G_{v}^{\prime}$ resulting from $G$ after the chordal completion on $G[M(v)]$ is triangulated.

As we mentioned in the introduction, the algorithm of Hoàng and Sritharan runs in $O\left(n^{3}\right)$ time, where $n$ is the number of vertices of the input graph. In order to be able to beat this, we need to avoid the chordal completion step. Indeed, we show how we can take advantage of the Algorithm PEO and of properties of the chordal completion in order to compute all necessary information without actually performing the chordal completion. In particular, we prove that the following results hold:

Lemma 3.1 Let $G$ be a graph, $v$ a vertex of $G$, and $\left(y_{1}, y_{2}, \ldots, y_{k}\right)$ a DegMN-ordering of the nonneighbors $M(v)$ of $v$ in $G$. Moreover, let $G_{v}^{\prime}$ be the graph resulting from $G$ after the chordal completion on $G[M(v)]$ with respect to the DegMN-ordering $\left(y_{1}, y_{2}, \ldots, y_{k}\right)$ and let $\sigma=\left(y_{1}, y_{2}, \ldots, y_{k}, x_{1}\right.$, $\left.x_{2}, \ldots, x_{\operatorname{deg}(v)}, v\right)$ where $x_{1}, x_{2}, \ldots, x_{\operatorname{deg}(v)}$ is an arbitrary ordering of the neighbors of $v$ in $G$. If Algorithm PEO $\left(G_{v}^{\prime}, \sigma\right)$ returns "false" while processing vertex $y_{i} \in M(v)$, then $A\left(y_{i}\right)-N\left(y_{i}\right) \subseteq N(v)$.

Proof: Since the Algorithm PEO returns "false" while processing vertex $y_{i} \in M(v)$, then $A\left(y_{i}\right)-$ $N\left(y_{i}\right) \neq \emptyset$. Suppose that there exists a vertex $y_{j} \in M(v)$ belonging to $A\left(y_{i}\right)-N\left(y_{i}\right)$. The vertex $y_{j}$ was added to $A\left(y_{i}\right)$ at $\operatorname{Step} 7$ of a prior iteration of the for-loop, say, while processing vertex $y_{\ell}$. It follows that $\sigma^{-1}\left(y_{\ell}\right)<\sigma^{-1}\left(y_{i}\right)<\sigma^{-1}\left(y_{j}\right)$, and $y_{i}, y_{j} \in N\left(y_{\ell}\right)$. Since $y_{j} \notin N\left(y_{i}\right)$, we have that $y_{\ell}$ is not simplicial in $G_{v}^{\prime}\left[\left\{y_{\ell}, y_{\ell+1}, \ldots, y_{k}\right\}\right]$; a contradiction to the definition of $G_{v}^{\prime}$.

Lemma 3.2 Let $G_{v}^{\prime}$ and $\sigma$ be as in the statement of Lemma 3.1 The vertex $v$ belongs to a $C_{5}$ or is the top of a house in the graph $G_{v}^{\prime}$ if and only if Algorithm PEO $\left.G_{v}^{\prime}, \sigma\right)$ returns "false" while processing vertex $z$, where $z \in M(v)$.

Proof: $(\Longleftarrow)$ The Algorithm $\operatorname{PEO}\left(G_{v}^{\prime}, \sigma\right)$ returns "false" while processing vertex $z$ only if at Step 8 there exists a vertex $x \in A(z)-N(z)$. From Lemma 3.1 we have that $A(z)-N(z) \subseteq N(v)$; thus, $x \in N(v)$. The vertex $x$ was added to $A(z)$ at Step 7 of a prior iteration of the for-loop, say, while processing vertex $y$; then, $\sigma^{-1}(y)<\sigma^{-1}(z)<\sigma^{-1}(x)$, and $y z \in E\left(G_{v}^{\prime}\right)$ and $x y \in E\left(G_{v}^{\prime}\right)$. Moreover, since $z \in M(v)$ and $x \notin N(z)$, we have that $y \in M(v)$ and $x z \notin E\left(G_{v}^{\prime}\right)$. Since $\sigma^{-1}(y)<\sigma^{-1}(z)$, the definition of the

(i) A building is a graph on vertices $v_{1}, v_{2}, \ldots, v_{p}$, where $p \geq 6$, and edges $v_{1} v_{p}, v_{2} v_{p}$, and $v_{i} v_{i+1}$ for $i=1,2, \ldots, p-1$; the vertex $v_{1}$ is called the top of the building. 
DegMN-ordering implies that $n(v, y) \leq n(v, z)$; because $x y \in E\left(G_{v}^{\prime}\right)$ and $x z \notin E\left(G_{v}^{\prime}\right)$, there exists a vertex $x^{\prime} \in N(v)$ such that $x^{\prime} z \in E\left(G_{v}^{\prime}\right)$ and $x^{\prime} y \notin E\left(G_{v}^{\prime}\right)$. But then, the vertices $v, x, x^{\prime}, y, z$ induce either a $C_{5}$ or a house: if $x x^{\prime} \notin E\left(G_{v}^{\prime}\right)$ then $v$ belongs to a $C_{5}$; otherwise, $v$ is the top of a house.

$\left(\Longrightarrow\right.$ ) Among the $C_{5}$ s of $G_{v}^{\prime}$ to which $v$ belongs and the houses of $G_{v}^{\prime}$ with $v$ as the top vertex, consider the $C_{5}$ or house whose vertices, say, $y$ and $z$, that belong to $M(v)$ are such that the quantity $\left|\sigma^{-1}(y)-\sigma^{-1}(z)\right|$ is minimized. Let $x, x^{\prime}$ be the remaining two vertices of the $C_{5}$ or house, where $x, x^{\prime} \in N(v), x y \in$ $E\left(G_{v}^{\prime}\right), x z \notin E\left(G_{v}^{\prime}\right), x^{\prime} z \in E\left(G_{v}^{\prime}\right)$, and $x^{\prime} y \notin E\left(G_{v}^{\prime}\right)$, and suppose without loss of generality that $\sigma^{-1}(y)<\sigma^{-1}(z)$ (see Figure 4 4 a); the dotted edge indicates a potential edge of $G$ ).

Next, we show that $z$ is the leftmost among the neighbors of $y$ that are to the right of $y$ (with respect to $\sigma)$ in $G_{v}^{\prime}$. Suppose for contradiction that the leftmost among these neighbors is $w \neq z$. Then, $\sigma^{-1}(y)<$ $\sigma^{-1}(w)<\sigma^{-1}(z)$, and since $y w \in E\left(G_{v}^{\prime}\right)$ and $y z \in E\left(G_{v}^{\prime}\right)$, the definition of the graph $G_{v}^{\prime}$ implies that $w z \in E\left(G_{v}^{\prime}\right)$. Additionally, if $x w \notin E\left(G_{v}^{\prime}\right)$, then due to the ordering $\sigma, n(v, y) \leq n(v, w)$, which (because $x y \in E\left(G_{v}^{\prime}\right)$ ) implies that there exists a vertex $p \in N(v)$ such that $p w \in E\left(G_{v}^{\prime}\right)$ and $p y \notin E\left(G_{v}^{\prime}\right)$; but then, the vertices $v, x, y, w, p$ induce a $C_{5}$ or a house to which $v$ belongs and $\left|\sigma^{-1}(y)-\sigma^{-1}(w)\right|<\left|\sigma^{-1}(y)-\sigma^{-1}(z)\right|$, in contradiction to the minimality of the $C_{5}$ or house induced by $v, x, y, z, x^{\prime}$. Thus, $x w \in E\left(G_{v}^{\prime}\right)$. Then, if $x^{\prime} w \notin E\left(G_{v}^{\prime}\right)$, the vertices $v, x, w, z, x^{\prime}$ induce a $C_{5}$ or a house to which $v$ belongs and $\left|\sigma^{-1}(w)-\sigma^{-1}(z)\right|<\left|\sigma^{-1}(y)-\sigma^{-1}(z)\right|$, in contradiction to the minimality of the $C_{5}$ or house induced by $v, x, y, z, x^{\prime}$. If however $x^{\prime} w \in E\left(G_{v}^{\prime}\right)$ then, because $n(v, w) \leq n(v, z)$ (since $\left.\sigma^{-1}(w)<\sigma^{-1}(z)\right)$ and because $x w \in E\left(G_{v}^{\prime}\right)$ whereas $x z \notin E\left(G_{v}^{\prime}\right)$, there exists a vertex $q \in N(v)$ such that $q z \in E\left(G_{v}^{\prime}\right)$ and $q w \notin E\left(G_{v}^{\prime}\right)$; but then, the vertices $v, x^{\prime}, w, z, q$ induce a $C_{5}$ or a house to which $v$ belongs and $\left|\sigma^{-1}(w)-\sigma^{-1}(z)\right|<\left|\sigma^{-1}(y)-\sigma^{-1}(z)\right|$, in contradiction again to the minimality of the $C_{5}$ or house induced by $v, x, y, z, x^{\prime}$. Therefore, $w=z$. Then, while processing vertex $y$, the Algorithm PEO includes vertex $x$ in $X$ in Step 4 and later in Step 7 adds $x$ in $A(z)$; then, while processing vertex $z$, the Algorithm PEO detects that $A(z)-N(z) \neq \emptyset$ since $x \notin N(z)$, and returns "false."

Lemma 3.1 implies that, while running $\operatorname{Algorithm} \operatorname{PEO}\left(G_{v}^{\prime}, \sigma\right)$, it suffices to collect in the set $X$ (Step 4) only the common neighbors of $u$ and $v$; in turn, Lemma 3.2 implies that it suffices to execute the for-loop of Steps 28 only for the non-neighbors of $v$.

Additionally, since neither the graph $G$ nor the ordering $\sigma$ changes during the execution of the Algorithm PEO, then, for each vertex $u$ of $G$, we can precompute the corresponding vertex $w$ (see Step 6). In fact, in light of Lemma 3.2, we can precompute an array Next_Neighbor ${ }_{G^{\prime}[M(v)], \sigma_{v}}[]$, such that for each vertex $u \in M(v)$, the entry $N_{\text {ext_Neighbor }}{ }_{G^{\prime}[M(v)], \sigma_{v}}[u]$ is equal to the leftmost among the neighbors of $u$ that are to the right of $u$ (with respect to a DegMN-ordering $\sigma_{v}$ of the non-neighbors $M(v)$ of $v$ in $G)$ in the chordal completion $G^{\prime}[M(v)]$ of the subgraph $G[M(v)]$. Note that a vertex $u \in M(v)$ need not have any neighbors among the vertices that follow it in $\sigma_{v}$; in such a case, the entry Next_Neighbor ${ }_{G^{\prime}[M(v)], \sigma_{v}}[u]$ does not get assigned a value, yet, since the for-loop (Steps 2 8 ) of Algorithm PEO will only be executed for the non-neighbors of $v$ because of Lemma 3.2, we do not need to update any set $A()$.

Based on the above, we obtain the Algorithm Not-in-HHB, presented in Figure 3, which takes as input a graph $G$ and a vertex $v$ of $G$, and returns "true" if and only if the vertex $v$ does not belong to a hole, and it is not the top of a house or a building in $G$. The correctness of Algorithm Not-in-HHB is established in the following theorem.

Theorem 3.1 Algorithm Not-in-HHB(G,v) returns "false" if and only if the vertex $v$ belongs to a hole or is the top of a house or a building in $G$. 
Algorithm Not-in- $H H B(G, v)$

1. Compute a DegMN-ordering $\sigma_{v}=\left(y_{1}, y_{2}, \ldots, y_{k}\right)$ of the non-neighbors of $v$ in the graph $G$;

compute the array Next_Neighbor $G_{G^{\prime}[M(v)], \sigma_{v}}$ [ ] for the chordal completion $G^{\prime}[M(v)]$ of the subgraph $G[M(v)]$ with respect to the ordering $\sigma_{v}$;

for each non-neighbor $u$ of $v$ do

$$
A(u) \leftarrow \emptyset ;
$$

2. for $i \leftarrow 1$ to $k$ do

3. $u \leftarrow \sigma_{v}(i)$;

4. $\quad X \leftarrow N(u) \cap N(v) ; \quad\left\{\right.$ note: $\forall x \in X$, u precedes $x$ in the ordering $\left.\sigma_{v}\right\}$

5. if $X \neq \emptyset$ and the entry $N_{\text {ext_Neighbor }}{ }_{G^{\prime}}[M(v)], \sigma_{v}[u]$ has been assigned a value

6. $\quad$ then $w \leftarrow N e x$ N_Neighbor $_{G^{\prime}[M(v)], \sigma_{v}}[u] ; \quad\{$ note: $w \in M(v)\}$

7. $\quad$ concatenate $X$ to $A(w) ; \quad\{$ note: $w \notin X\}$

8. if $A(u)-N(u) \neq \emptyset$ then return("false");

9. return("true");

Fig. 3: The algorithm for determining whether a vertex $v$ belongs to a hole or is the top of a house or a building.

Proof: ( $\Longrightarrow$ ) Suppose that the algorithm returns "false" while processing vertex $z \in M(v)$ (i.e, when $\left.i=\sigma_{v}^{-1}(z)\right)$. This happens only if at Step 8 of the current iteration of the for-loop there exists a vertex $x \in A(z)-N(z)$. Then, from Lemma 3.2 we have that $A(z)-N(z) \subseteq N(v)$, which implies that $x \in$ $N(v)$ and $x z \notin E(G)$. The vertex $x$ was added to $A(z)$ at Step 7 of a prior iteration, say, while processing vertex $y$; thus, $x y \in E(G)$ and $z=N e x t_{-} N_{\text {eighbor }}{ }_{G^{\prime}[M(v)], \sigma_{v}}[y]$ which implies that $\sigma_{v}^{-1}(y)<\sigma_{v}^{-1}(z)$ (and thus $y \in M(v)$ ), and $y z \in E\left(G^{\prime}[M(v)]\right)$. As in the proof of Lemma 3.2, we can show that there exists a vertex $x^{\prime}$ of $G$ such that $x^{\prime} \in N(v), x^{\prime} z \in E(G)$, and $x^{\prime} y \notin E(G)$ (see Figure 4(a), where the dotted edge indicates a potential edge of $G$ ). For the vertices $y, z$ (which are adjacent in $G^{\prime}[M(v)]$ ), we distinguish two cases:

$y z \in E(G)$. Then, if $x x^{\prime} \notin E(G)$, the vertex set $\left\{v, x, y, z, x^{\prime}\right\}$ induces a hole (in fact, a $C_{5}$ ), otherwise it induces a house with vertex $v$ at the top.

$y z \notin E(G)$. Then, because $z=$ Next_Neighbor $_{G^{\prime}[M(v)], \sigma_{v}}[y]$ (i.e., the vertices $y, z$ are adjacent in the chordal completion $G^{\prime}[M(v)]$ ), Lemma 2 of [9] implies that the graph $G$ has an induced path on at least three vertices connecting $y$ and $z$ all of whose vertices are in $M(v)$ and such that for each vertex $w$ of the path other than $y, z$, it holds that $N(w) \cap N(v) \subseteq N(y) \cap N(v)$. Let $a_{1} a_{2} \cdots a_{q}$ be a chordless such path where $a_{1}=y, a_{q}=z$, and $q \geq 3$; then, $a_{i} \in M(v)$ and $N\left(a_{i}\right) \cap N(v) \subseteq$ $N(y) \cap N(v)$, for all $1<i<q$ (see Figure $4(\mathrm{~b}$ ), where the dashed edge indicates an edge in $\left.E\left(G^{\prime}[M(v)]\right)-E(G)\right)$. Since $x^{\prime} \notin N(y)$, this implies that $x^{\prime} \notin N\left(a_{i}\right)$ for all $i=1,2, \ldots, q-1$. 


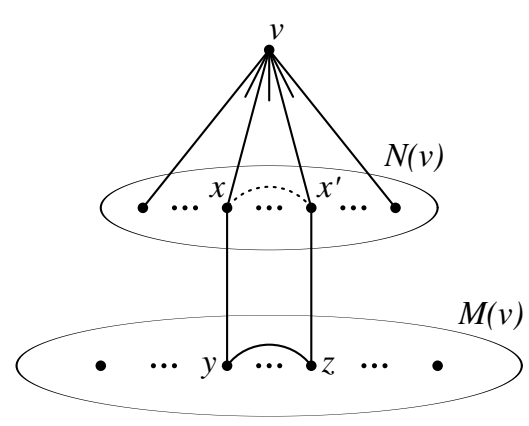

(a)

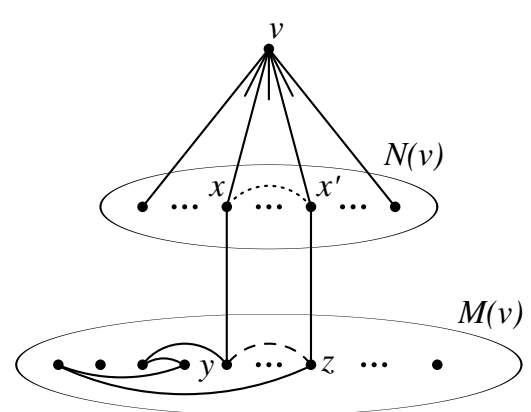

(b)

Fig. 4

Let $p=\max \left\{i \mid x \in N\left(a_{i}\right)\right\} ; p$ is well defined since $x \in N\left(a_{1}\right)$, and $p<q$ since $x \notin N\left(a_{q}\right)$ 一recall that $a_{1}=y, a_{q}=z, x y \in E(G)$, and $x z \notin E(G)$. Then, the vertices $a_{p+1}, \ldots, a_{q-1}$ are not adjacent to $x$ or $x^{\prime}$, so that if $x x^{\prime} \notin E(G)$, the vertices $v, x, a_{p}, \ldots, a_{q-1}, z, x^{\prime}$ induce a hole in $G$, whereas if $x x^{\prime} \in E(G)$ they induce a house or a building with $v$ at its top.

In both cases, the vertex $v$ belongs to a hole or is the top of a house or a building in the graph $G$.

( $\Longleftarrow$ ) Suppose that the vertex $v$ belongs to a hole or is the top of a house or a building in $G$. If $v$ belongs to a $C_{5}$ or is the top of a house, let $v, x, y, z, x^{\prime}$ be the vertices inducing the $C_{5}$ or house $\left(x, x^{\prime} \in\right.$ $N(v), y, z \in M(v), x y \in E(G)$, and $x^{\prime} z \in E(G)$ ); if $v$ belongs to a hole on more than 5 vertices or is the top of a building, we consider such a hole or building on the fewest vertices, and let them be $v, x, y, a_{1}, \ldots, a_{p}, z, x^{\prime}\left(x, x^{\prime} \in N(v), y, a_{1}, \ldots, a_{p}, z \in M(v), x y \in E(G)\right.$, and $x^{\prime} z \in E(G)$ ). Moreover, suppose without loss of generality that in either case $\sigma_{v}^{-1}(y)<\sigma_{v}^{-1}(z)$. In case $v$ belongs to a hole on $\geq 6$ vertices or is the top of a building, we can show that all the $a_{i}$ s precede both $y$ and $z$ in the ordering $\sigma_{v}$ : if any $a_{i}$ appears between $y$ and $z$, then the definition of the DegMN-ordering $\sigma_{v}$ implies that there exists vertex $x^{\prime \prime} \in N(v)$ such that $x^{\prime \prime} \in N\left(a_{i}\right)-N(y)$ (recall that $x y \in E(G)$ and $x a_{i} \notin E(G)$ ); then the vertices $v, x, y, a_{1}, \ldots, a_{i}, x^{\prime \prime}$ induce a hole or a building on fewer vertices. Then, this property of the $a_{i} \mathrm{~s}$ and Lemma 3.3 imply that $y z \in E\left(G^{\prime}[M(v)]\right)$.

From the above, we conclude that, in any case, the vertices $y, z$ are adjacent in $G^{\prime}[M(v)]$; then, we show that there exists a sequence $y=b_{1}, b_{2}, \ldots, b_{q}=z$ of non-neighbors of $v$ in $G$ such that $b_{i}=$ $N_{\text {Next_Neighbor }}{ }_{G^{\prime}[M(v)], \sigma_{v}}\left[b_{i-1}\right]$ for $2 \leq i \leq q$ (note that $b_{i}=$ Next_Neighbor $_{G^{\prime}[M(v)], \sigma_{v}}\left[b_{i-1}\right]$ implies that $\sigma_{v}^{-1}\left(b_{i-1}\right)<\sigma_{v}^{-1}\left(b_{i}\right)$ and $\left.b_{i-1} b_{i} \in E\left(G^{\prime}[M(v)]\right)\right)$. The existence of such a sequence is shown as follows: if $z=N e x t_{-} N e i g h b o r_{G^{\prime}[M(v)], \sigma_{v}}[y]$, then the sequence is precisely $y, z$; otherwise, if $b_{2}=$ Next_Neighbor $G_{G^{\prime}[M(v)], \sigma_{v}}[y]$, then $y b_{2} \in E\left(G^{\prime}[M(v)]\right)$ which from the fact that $y z \in E\left(G^{\prime}[M(v)]\right)$ and from the definition of $G^{\prime}[M(v)]$ implies that $b_{2} z \in E\left(G^{\prime}[M(v)]\right)$; next, we repeat the above argument for $b_{2}$ in the place of $y$, and so on so forth; since there is a finite number of vertices between $y$ and $z$ in $\sigma_{v}$, eventually, we will find a vertex $b_{q-1}$ such that $z=N e x \operatorname{Ne}_{-} \operatorname{Neighbor}_{G^{\prime}[M(v)], \sigma_{v}}\left[b_{q-1}\right]$. Let $r=\max \left\{i \mid x \in N\left(b_{i}\right)\right\} ; r$ is well defined since $x \in N\left(b_{1}\right)$, and $r<q$ since $x \notin N\left(b_{q}\right)$ recall that $b_{1}=y$ and $x y \in E(G)$, and $b_{q}=z$ and $x z \notin E(G)$. Since $x \in N\left(b_{r}\right)$ and $b_{r+1}=$ Next_Neighbor ${ }_{G^{\prime}[M(v)], \sigma_{v}}\left[b_{r}\right]$, the processing of vertex $b_{r}$ will result in the addition of vertex $x$ in the set $A\left(b_{r+1}\right)$; then, the Algorithm Not-in-HHB will return "false" when it processes vertex $b_{r+1}$, because 
Algorithm Recognize-HH-free

1. for each vertex $v$ of the input graph $G$ do

if Algorithm Not-in- $\operatorname{HHB}(G, v)$ returns "false"

then return("the graph is not HH-free"); $\quad\{G$ contains a house or a hole $\}$

2. return("the graph is HH-free");

Fig. 5: The algorithm for determining whether a graph $G$ is HH-free.

$x \notin N\left(b_{r+1}\right)$, if not earlier.

In light of Theorem 3.1 and by observing that a building contains a hole, we obtain the very simple $\mathrm{HH}$-free graph recognition algorithm that is given in Figure 5

\subsection{Computation of the values of Next_Neighbor ${ }_{G^{\prime}[S], \sigma}[]$.}

Let $G$ be a graph, $S$ a subset of the vertex set of $G, \sigma$ an ordering of the vertices in $S$, and let $G^{\prime}[S]$ be the chordal completion of the subgraph $G[S]$ with respect to $\sigma$. For each vertex $x \in S$, we want to determine the leftmost among the neighbors of $x$ that are to the right of $x$ (w.r.t. $\sigma$ ) in $G^{\prime}[S]$. In order to avoid computing the graph $G^{\prime}[S]$, we take advantage of the following property of the chordal completion:

Lemma 3.3 Let $G$ be a graph, let $\left(v_{1}, v_{2}, \ldots, v_{k}\right)$ be an ordering of a vertex set $S \subseteq V(G)$, and let $G^{\prime}$ be the graph resulting from the subgraph $G[S]$ after we had applied chordal completion on it with respect to the ordering of the vertices in $S$ (i.e., after having added edges so that, for all $i=1,2, \ldots, k$, vertex $v_{i}$ is simplicial in the subgraph induced by the vertices $\left.v_{i}, v_{i+1}, \ldots, v_{k}\right)$. Then, the graph $G^{\prime}$ contains the edge $v_{r} v_{j}$, where $r<j$, if and only if there exists an edge $v_{i} v_{j}$ in $G$ such that $i \leq r$ and the vertices $v_{i}, v_{r}$ belong to the same connected component of the subgraph of $G$ induced by the vertices $v_{1}, v_{2}, \ldots, v_{i}, \ldots, v_{r}$.

Proof: $(\Longrightarrow)$ Suppose that $v_{r} v_{j} \in E\left(G^{\prime}\right)$. We will show the following:

Proposition. If $v_{r} v_{j} \in E\left(G^{\prime}\right)$, then there exists a vertex $v_{i}$, where $i \leq r$, such that $v_{i} v_{j}$ is an edge of $G$ and the vertices $v_{i}, v_{r}$ belong to the same connected component of the subgraph $G\left[\left\{v_{1}, v_{2}, \ldots, v_{i}, \ldots, v_{r}\right\}\right]$.

We use induction on $r$.

Basis: $r=1$. Then, since $v_{1} v_{j} \in E\left(G^{\prime}\right)$, it can only be that $v_{1} v_{j} \in E(G)$ and the proposition holds with $v_{i}$ being $v_{1}$.

Inductive hypothesis: Suppose that the proposition holds for all $r<\hat{r}$, where $\hat{r} \geq 2$.

Inductive step: We show that the proposition holds for $r=\hat{r}$.

Hence, suppose that $v_{\hat{r}} v_{j} \in E\left(G^{\prime}\right)$. If $v_{\hat{r}} v_{j}$ is an edge of $G$, then the proposition clearly holds with $v_{i}$ being $v_{\hat{r}}$. Otherwise, the edge $v_{\hat{r}} v_{j}$ must have been added while making a vertex $v_{t}$ simplicial; 
then, $t<\hat{r}<j$ and $v_{t} v_{\hat{r}}, v_{t} v_{j} \in E\left(G^{\prime}\right)$. Since $v_{t} v_{\hat{r}} \in E\left(G^{\prime}\right)$ and $t<\hat{r}$, then by the inductive hypothesis, there exists a vertex $v_{p}$, where $p \leq t<\hat{r}$, such that $v_{p} v_{\hat{r}}$ is an edge of $G$ and $v_{p}, v_{t}$ belong to the same connected component of $G\left[\left\{v_{1}, v_{2}, \ldots, v_{t}\right\}\right]$. Similarly, by the inductive hypothesis for the edge $v_{t} v_{j}$ of $G^{\prime}$, there exists a vertex $v_{q}$, where $q \leq t<j$, such that $v_{q} v_{j}$ is an edge of $G$ and $v_{q}, v_{t}$ belong to the same connected component of $G\left[\left\{v_{1}, v_{2}, \ldots, v_{t}\right\}\right]$. Therefore, the vertices $v_{p}, v_{q}$ belong to the same connected component of $G\left[\left\{v_{1}, v_{2}, \ldots, v_{t}\right\}\right]$. Then, the existence of the edge $v_{p} v_{\hat{r}}$ in $G$ and the fact that $p \leq t<\hat{r}$ imply that $v_{p}, v_{\hat{r}}$ belong to the same connected component of $G\left[\left\{v_{1}, v_{2}, \ldots, v_{\hat{r}}\right\}\right]$; moreover, since $v_{p} v_{j}$ is an edge of $G$ and $p \leq t<\hat{r}<j$, the proposition holds with $v_{i}$ being $v_{p}$.

The induction then implies that the proposition holds for all edges $v_{r} v_{j} \in E\left(G^{\prime}\right)$, where $r<j$.

( $\Longleftarrow$ ) Suppose now that for vertices $v_{r}, v_{j}$ of $G$, where $r<j$, there exists a vertex $v_{i}$, where $i \leq r$, such that $v_{i} v_{j}$ is an edge of $G$ and the vertices $v_{i}, v_{r}$ belong to the same connected component of the subgraph $G\left[\left\{v_{1}, v_{2}, \ldots, v_{r}\right\}\right]$. We will show that $v_{r} v_{j} \in E\left(G^{\prime}\right)$.

If $v_{r} v_{j} \in E(G)$, then clearly $v_{r} v_{j} \in E\left(G^{\prime}\right)$. Suppose now that $v_{r} v_{j} \notin E(G)$. Since the vertices $v_{i}, v_{r}$ belong to the same connected component of $G\left[\left\{v_{1}, v_{2}, \ldots, v_{r}\right\}\right]$, there exists a simple path $v_{r} v_{p_{1}} v_{p_{2}} \cdots v_{p_{\ell}} v_{i}$ from $v_{r}$ to $v_{i}$ in $G\left[\left\{v_{1}, v_{2}, \ldots, v_{r}\right\}\right]$; then, clearly, $p_{t}<r$ for all $t=1,2, \ldots, \ell$. Since $v_{i} v_{j}$ is an edge of $G$, then $v_{r} v_{p_{1}} v_{p_{2}} \cdots v_{p_{\ell}} v_{i} v_{j}$ is a simple path from $v_{r}$ to $v_{j}$ in $G$, and hence in $G^{\prime}$. For ease of notation, let us set $v_{p_{0}}=v_{r}, v_{p_{\ell+1}}=v_{i}$, and $v_{p_{\ell+2}}=v_{j}$, so that the path from $v_{r}$ to $v_{j}$ becomes $v_{p_{0}} v_{p_{1}} v_{p_{2}} \cdots v_{p_{\ell+1}} v_{p_{\ell+2}}$. Let $p_{s}$ be the minimum among $p_{1}, p_{2}, \ldots p_{\ell+1}$, i.e., $v_{p_{s}}$ is the leftmost (with respect to $\sigma$ ) vertex among $v_{p_{1}}, v_{p_{2}}, \ldots v_{p_{\ell+1}}$; then, since for all $t=1,2, \ldots, \ell+1$ it holds that $p_{t}<r<j$, we have that $p_{s}<p_{s-1}$ and $p_{s}<p_{s+1}$. The definition of the graph $G^{\prime}$ implies that $v_{p_{s}}$ is simplicial in $G^{\prime}\left[\left\{v_{p_{s}}, v_{p_{s}+1}, \ldots, v_{k}\right\}\right]$; since $v_{p_{s}}$ is adjacent in $G^{\prime}$ to $v_{p_{s-1}}$ and to $v_{p_{s+1}}, G^{\prime}$ contains the edge $v_{p_{s-1}} v_{p_{s+1}}$. Thus, $v_{p_{0}} v_{p_{1}} \cdots v_{p_{s-1}}$ $v_{p_{s+1}} \cdots v_{p_{\ell+2}}$ is a simple path in $G^{\prime}$ from $v_{r}$ to $v_{j}$, i.e., we obtained a path in $G^{\prime}$ from $v_{r}$ to $v_{j}$ where the leftmost vertex $v_{p_{s}}$ has been removed. The process of removing the leftmost (w.r.t. $\sigma$ ) vertex among the vertices of the path can be repeated over and over, and in each case we obtain a shorter simple path in $G^{\prime}$ from $v_{r}$ to $v_{j}$. Since $p_{t}<r<j$ for all $t=1,2, \ldots, \ell+1$, eventually all the vertices $v_{p_{1}}, v_{p_{2}}, \ldots, v_{p_{\ell+1}}$ will be removed and we will have that $v_{p_{0}} v_{p_{\ell+2}}=v_{r} v_{j}$ is a path (= edge) in $G^{\prime}$, as desired.

We note that the above lemma implies Lemma 2 of [9] as a corollary. Lemma 3.3 implies that for the computation of the value Next_Neighbor ${ }_{G^{\prime}[S], \sigma}\left[v_{r}\right]$, where $\sigma=\left(v_{1}, v_{2}, \ldots, v_{k}\right)$, it suffices to find the leftmost (w.r.t. $\sigma$ ) vertex among $v_{r+1}, v_{r+2}, \ldots, v_{k}$ which is adjacent in $G$ to a vertex in the connected component of $G\left[\left\{v_{1}, v_{2}, \ldots, v_{r}\right\}\right]$ to which $v_{r}$ belongs. This can be efficiently done by processing the vertices $v_{1}, v_{2}, \ldots, v_{k}$ in order from $v_{1}$ to $v_{k}$. In detail, the algorithm to compute the contents of the array $N_{1} t_{-} N_{\text {eighbor }}{ }_{G^{\prime}[S], \sigma}[]$ is presented in Figure 6, note that if a vertex $v_{i} \in S$ has no neighbors in $G^{\prime}\left[\left\{v_{i}, \ldots, v_{k}\right\}\right]$, then the entry Next_Neighbor $_{G^{\prime}[S], \sigma}\left[v_{i}\right]$ is not assigned a value.

It is important to observe the following:

Observation 3.1 At the completion of the processing of vertex $v_{j}$ in Algorithm Compute-Next_Neighbor, the sets of vertices maintained by the algorithm are in a bijection with the connected components of $G\left[\left\{v_{1}, v_{2}, \ldots, v_{j}\right\}\right]$;

The observation follows from the fact that while processing $v_{j}$, we consider the edges $v_{i} v_{j}$ where $i<j$, and we union the set containing $v_{j}$ (which has vertex $v_{j}$ as its rightmost vertex with respect to $\sigma$ ) to another set iff $v_{j}$ is adjacent to a vertex in that set. The correctness of Algorithm Compute-Next_Neighbor is established in the following lemma. 
Algorithm Compute-Next_Neighbor $(G, S, \sigma)$

1. $\left\{\right.$ let $\sigma=\left(v_{1}, v_{2}, \ldots, v_{k}\right)$ be the given ordering of the vertices in $\left.S\right\}$ make a set containing the vertex $v_{1}$;

2. for $j=2,3, \ldots, k$ do

3. $\quad$ make a set containing the vertex $v_{j}$;

4. for each edge $v_{i} v_{j}$ of $G$, where $i<j$, do

5. $\quad v_{r} \leftarrow$ the rightmost (w.r.t. $\sigma$ ) vertex in the set to which $v_{i}$ belongs;

6. $\quad$ if $v_{r} \neq v_{j}$

7. then $\quad\left\{v_{i}\right.$ and $v_{j}$ belong to different sets $\}$

8. $\quad$ Next_Neighbor ${ }_{G^{\prime}[S], \sigma}\left[v_{r}\right] \leftarrow v_{j}$;

9. $\quad$ union the sets to which $v_{i}$ and $v_{j}$ belong;

Fig. 6: The algorithm for computing the contents of the array $N e x t_{-} N e i g h b o r_{G^{\prime}[S], \sigma}[]$.

Lemma 3.4 The Algorithm Compute-Next_Neighbor correctly computes the values of the entries Next_Neighbor $_{G^{\prime}[S], \sigma}\left[v_{i}\right]$ for all the vertices $v_{i} \in S$.

Proof: First, we show that, for a vertex $v_{r} \in S$, the Algorithm Compute-Next_Neighbor assigns a value to the entry Next_Neighbor ${ }_{G^{\prime}[S], \sigma}\left[v_{r}\right]$ if and only if the vertex $v_{r}$ is adjacent in the graph $G^{\prime}[S]$ to a vertex in $\left\{v_{r+1}, v_{r+2}, \ldots, v_{k}\right\}$.

The entry Next_Neighbor ${ }_{G^{\prime}[S], \sigma}\left[v_{r}\right]$ is assigned a value if, during the processing of a vertex $v_{j}$, where $j>r$, there exists an edge in $G$ connecting $v_{j}$ to a vertex $v_{i}$, and $v_{r}$ is the rightmost (w.r.t. $\sigma$ ) vertex among the vertices in the set containing $v_{i}$ (Step 5 of Algorithm Compute-Next_Neighbor). Since the vertices $v_{i}$ and $v_{r}$ belong to the same set, which corresponds to the same connected component of the subgraph $G\left[\left\{v_{1}, v_{2}, \ldots, v_{j-1}\right\}\right]$ (Observation 3.1), and since $v_{r}$ is the rightmost vertex in the set, then $v_{i}, v_{r}$ belong to the same connected component of $G\left[\left\{v_{1}, v_{2}, \ldots, v_{r}\right\}\right]$. Due to this and due to the fact that $v_{r} v_{j} \in E(G)$, where $r<j$, Lemma 3.3 implies that the chordal completion $G^{\prime}[S]$ of the subgraph $G[S]$ of $G$ induced by the elements of $S$ contains the edge $v_{r} v_{j}$.

Suppose now that a vertex $v_{i}$ is adjacent in $G^{\prime}[S]$ to a vertex in $\left\{v_{i+1}, v_{i+2}, \ldots, v_{k}\right\}$; we show that the Algorithm Compute-Next_Neighbor assigns a value to the entry Next_Neighbor $_{G^{\prime}[S], \sigma}\left[v_{i}\right]$. Let $v_{s}$ be the leftmost (w.r.t. $\sigma$ ) vertex among the vertices in $\left\{v_{i+1}, v_{i+2}, \ldots, v_{k}\right\}$ which are adjacent in $G$ to a vertex in the connected component of $G\left[\left\{v_{1}, v_{2}, \ldots, v_{i}\right\}\right]$ to which $v_{i}$ belongs. We show that right before processing $v_{s}$, the rightmost (w.r.t. $\sigma$ ) vertex in the set to which $v_{i}$ belongs is precisely $v_{i}$. Suppose for contradiction that this is not the case and let $v_{s^{\prime}}$ be the rightmost vertex, where $s^{\prime}>i$. Then, there exists a simple path in $G$ from $v_{i}$ to $v_{s^{\prime}}$; let it be $v_{p_{0}} v_{p_{1}} v_{p_{2}} \cdots v_{p_{h}}$, where $v_{p_{0}}=v_{i}$ and $v_{p_{h}}=v_{s^{\prime}}$. Clearly, $p_{t}<s^{\prime}$ for all $t=1,2, \ldots, h-1$. Let $\hat{t}=\min \left\{t \in\{1,2, \ldots, h\} \mid p_{t}>i\right\} ; \hat{t}$ is well defined since $p_{h}=s^{\prime}>i$. Then, $v_{p_{t}} v_{p_{\hat{t}-1}} \in E(G)$ and since $v_{p_{0}} v_{p_{1}} \cdots v_{p_{\hat{t}-1}}$ is a path in $G$ and $p_{t} \leq i$ for all $t=0,1, \ldots, \hat{t}-1$, the vertices $v_{p_{\hat{t}-1}}$ and $v_{i}$ belong to the same connected component of $G\left[\left\{v_{1}, v_{2}, \ldots, v_{i}\right\}\right]$; however, these facts come to a contradiction with the definition of $v_{s}$, because $i<p_{\hat{t}}<s$. Therefore, right before 
processing vertex $v_{s}, v_{i}$ indeed is the rightmost (w.r.t. $\sigma$ ) vertex in the set to which it belongs, and thus the entry $N_{e x t}$ Neighbor $_{G^{\prime}[S], \sigma}\left[v_{i}\right]$ will be assigned a value (it will be set equal to $v_{s}$ during the processing of vertex $v_{s}$ ).

The proof will be complete if we show that, whenever the Algorithm Compute-Next_Neighbor assigns a value to an entry Next_Neighbor ${ }_{G^{\prime}[S], \sigma}\left[v_{r}\right]$, it assigns the correct value. Observe that whenever the algorithm executes an assignment Next_Neighbor ${ }_{G^{\prime}[S], \sigma}\left[v_{r}\right] \leftarrow v_{j}$, then $r<j$ and there exists an edge $v_{i} v_{j}$ in $G$ such that $i<j$ and the vertices $v_{i}, v_{j}$ belong to the same connected component of the subgraph $G\left[\left\{v_{1}, v_{2}, \ldots, v_{r}\right\}\right]$. Then, Lemma 3.3 implies that the chordal completion $G^{\prime}[S]$ of the subgraph $G[S]$ of $G$ induced by the elements of $S$ contains the edge $v_{r} v_{j}$. We need to show that $v_{r}$ is not adjacent in the chordal completion of $G[S]$ to any vertex $v_{t}$, where $r<t<j$. Suppose for contradiction that there existed such a vertex $v_{t}$. Then, by Lemma 3.3, there exists a vertex $v_{s}$ such that $s<r, v_{s} v_{t} \in E(G)$, and $v_{s}, v_{r}$ belong to the same connected component of $G\left[\left\{v_{1}, v_{2}, \ldots, v_{r}\right\}\right]$. But then, while processing the vertex $v_{t}, v_{t}$ will be included in the set containing $v_{r}$, in contradiction to the fact that, while processing $v_{j}$ (which is done after processing $v_{t}$ since $t<j$ ), the rightmost (w.r.t. $\sigma$ ) vertex in the set containing $v_{r}$ is precisely $v_{r}$; recall that $r<t$. Therefore, all the assignments to the entries of the array Next_Neighbor ${ }_{G^{\prime}[S], \sigma}[]$ are correct.

Time and Space Complexity. Let $m$ be the number of edges of the graph $G$; recall also that $|S|=k$. If we ignore the operations to process sets (i.e., make a set, union sets, or find the rightmost (w.r.t. $\sigma$ ) vertex in a set) while running Algorithm Compute-Next_Neighbor $(G, S, \sigma)$, then the rest of the execution of the algorithm takes $O(k+m)$ time. The sets are maintained by our algorithm in a fashion amenable for Union-Find operations, where additionally the representative of each set also carries a link to the node storing the rightmost (w.r.t. $\sigma$ ) vertex in the set. Then,

- making a set which contains a single vertex $v_{i}$ requires building the set and setting the rightmost (w.r.t. $\sigma$ ) vertex in the set to $v_{i}$;

- finding the rightmost (w.r.t. $\sigma$ ) vertex in the set, say, $A$, to which a vertex $v_{j}$ belongs, requires performing a Find operation to locate the representative of the set $A$, from which the rightmost vertex is obtained in constant time;

- unioning two sets requires constructing a single set out of the elements of the two sets, and updating the rightmost (w.r.t. $\sigma$ ) vertex information; since we always union a set with the set containing $v_{j}$, where $v_{j}$ is the rightmost vertex in any of the sets, then the rightmost vertex of the resulting set is $v_{j}$, and this assignment can be done in constant time per union.

As the Algorithm Compute-Next_Neighbor creates one set for each one of the vertices $v_{1}, v_{2}, \ldots, v_{k}$, it executes $k$ make-set operations; this also implies that the number of union operations is less than $k$. The number of times to find the rightmost (w.r.t. $\sigma$ ) vertex in a set is $O(m)$, since the algorithm executes one such operation for each edge of the subgraph $G[S]$. The sets may be represented as disjoint-set forests, in which case, the time to execute $p$ make-set, find, and union operations involving $q$ elements is $O(p \alpha(p, q))$ [17], where $\alpha($,$) denotes the very slowly growing functional inverse of Ackerman's function; since$ Algorithm Compute-Next_Neighbor performs $p$ operations, where $k \leq p=O(k+m)$, on $k$ elements, and the function $\alpha(p, q)$ decreases as the ratio $p / q$ increases, the use of disjoint-set forests implies a time complexity of $O(m \alpha(k, k))$. Alternatively, the sets may be represented by linked lists, and the time to 
execute $p$ make-set, find, and union operations involving $q$ elements is $O(p+q \log q)$ [3]; thus, in this case, the time complexity is $O(m+k \log k)$. In either case, the space required (in addition to the space needed to store the graph $G)$ is $O(k)$. Thus, the computation of the values of the array Next_Neighbor ${ }_{G^{\prime}[S], \sigma}[]$ for the $k$ elements of the set $S$ takes a total of $O(\min \{(k+m) \alpha(k, k), m+k \log k\})$ time and $O(k)$ space. Therefore, we have:

Lemma 3.5 Let $G$ be a graph on $m$ edges, $S$ a subset of vertices of $G$, and $\sigma$ an ordering of the elements of $S$. Algorithm Compute-Next_Neighbor $(G, S, \sigma)$ correctly computes the values of the entries of the array Next_Neighbor ${ }_{G^{\prime}[S], \sigma}[]$ in $O(\min \{(k+m) \alpha(k, k), m+k \log k\})$ time and $O(k)$ space, where $k$ is the cardinality of the set $S$.

\subsection{Complexity of the Algorithm Recognize-HH-free}

Let us assume that the graph $G$ has $n$ vertices and $m$ edges and that vertex $v$ of $G$ has $k$ non-neighbors in $G$. It is not difficult to see that the execution of the Algorithm Not-in-HHB $(G, v)$ for vertex $v$ takes $O(n+m)$ time and space plus the time and space needed for the computation of the entries of the array $N e x t_{-} N e i g h b o r_{G^{\prime}[M(v)], \sigma_{v}}[]$. For the latter computation, we run Algorithm Compute-Next_Neighbor on $G, M(v)$, and $\sigma_{v}$, which takes $O(\min \{(k+m) \alpha(k, k), m+k \log k\})=O(\min \{(n+m) \alpha(n, n), m+$ $n \log n\}$ ) time and $O(k)=O(n)$ space (Lemma 3.5p; note that the function $\alpha(i, i)$ increases as $i$ increases (see definition in [17]). Therefore, Algorithm Not-in-HHB $(G, v)$ takes $O(n+m+\min \{(n+$ $m) \alpha(n, n), m+n \log n\})=O(\min \{(n+m) \alpha(n, n), m+n \log n\})$ and $O(n+m)$ space. Hence, we have:

Theorem 3.2 Let $G$ be a graph on $n$ vertices and $m$ edges. Algorithm Not-in-HHB determines whether a vertex $v$ of $G$ belongs to a hole or is the top of a house or a building in $O(\min \{(n+m) \alpha(n, n)$, $m+n \log n\})$ time and $O(n+m)$ space.

The Algorithm Recognize-HH-free consists of applying the Algorithm Non-in-HHB on every vertex of the input graph $G$. If $G$ is connected, then $n=O(m)$ and processing all the vertices of $G$ takes $O\left(\sum_{v} \min \{m \alpha(n, n), m+n \log n\}\right)$ time and $O(n+m)$ space. If $G$ is not connected, then we compute its connected components [3] and work on each of them separately (note that a hole and a house are both connected); clearly, the subgraph of $G$ induced by each such component is connected and has $O(n)$ vertices and $O(m)$ edges. Then, the computation of the components and the processing of all the vertices of $G$ takes $O\left(n+m+\sum_{v} \min \{m \alpha(n, n), m+n \log n\}\right)$ total time and $O(n+m)$ space. Summarizing, we obtain the following corollary:

Corollary 3.1 Algorithm Recognize-HH-free determines whether a graph $G$ on $n$ vertices and $m$ edges contains a hole or a house (i.e., is not HH-free) in $O(n \min \{m \alpha(n, n), m+n \log n\})$ time and $O(n+m)$ space.

\subsection{Providing a Certificate}

The Algorithm Recognize-HH-free can be made to provide a certificate (a hole or a house) whenever it decides that the input graph $G$ is not $\mathrm{HH}$-free. In particular, we augment the Algorithm Not-in- $\operatorname{HHB}(G, v)$ (5) as follows:

- When processing vertex $u$, we add a reference to $u$ to each element of the set $X$ formed in Step 4, so that for each vertex $w$, each element of the set $A(w)$ carries a reference to the vertex during whose processing this element was added to $A(w)$ (see Step 7). 
Our approach follows the proof of Theorem 3.1. The Algorithm Recognize-HH-free answers that the graph $G$ is not $\mathrm{HH}$-free when a call to Algorithm Not-in- $\operatorname{HHB}(G, v)$ for a vertex $v$ returns "false," i.e., when in Step 8 the difference $A(u)-N(u)$ is non-empty for a non-neighbor $u$ of $v$ in $G$. Let $x \in N(v)$ be an element in $A(u)-N(u)$; the vertex $x$ is associated with a vertex $y \in M(v)$ during whose processing vertex $x$ was added to $A(u)$ (then, clearly, $u=N e x t$ Neighbor $\left._{G^{\prime}[M(v)], \sigma_{v}}[y]\right)$ (ii) Then, we can obtain a hole or a house of $G$ by doing the following:

1. We traverse the neighbors of vertex $u$ and find a vertex $x^{\prime}$ which is adjacent to $v$ and not adjacent to $y$; such a vertex always exists since $n(v, y) \leq n(v, u)$ (note that $y$ precedes $u$ in the DegMNordering $\sigma_{v}$ ) and $x \in N(y) \cap N(v)$ whereas $x \notin N(u) \cap N(v)$.

2. We consider the subgraph of $G$ induced by $u, y$ and the vertices preceding $y$ in $\sigma_{v}$, and we apply BFS on it starting at $y$ until $u$ is reached (note that $u$ will be eventually reached, because $u=$ Next_Neighbor $_{G^{\prime}[M(v)], \sigma_{v}}[y]$, and thus $y$ and $u$ belong to the same connected component of the subgraph of $G$ induced by $u, y$ and the vertices preceding $u$ in $\sigma_{v}$; see Lemma 3.3; let $a_{1} a_{2} \cdots a_{q}$, where $a_{1}=y$ and $a_{q}=u$, be the path in the BFS-tree from $y$ to $u$, which is thus chordless. Moreover, $\left\{a_{1}, a_{2}, \ldots, a_{q-1}\right\} \cap N\left(x^{\prime}\right)=\emptyset$ (otherwise, the algorithm would have exited while processing a vertex preceding $u$; see proof of Theorem 3.1.

3. We compute $p=\max \left\{i \mid x \in N\left(a_{i}\right)\right\}$. Then, if $x x^{\prime} \notin E(G)$, the vertices $v, x, a_{p}, \ldots, a_{q-1}, u, x^{\prime}$ induce a hole in $G$; if $x x^{\prime} \in E(G)$, the vertices $v, x, a_{p}, u, x^{\prime}$ induce a house in $G$ if $p=q-1$, whereas if $p \leq q-2$, the vertices $x, a_{p}, \ldots, a_{q-1}, u, x^{\prime}$ induce a hole.

The correctness of the computation follows from the proof of Theorem 3.1. Regarding the time and space complexity, we first note that the augmentation of the Algorithm Not-in-HHB does not asymptotically increase the time and space complexity of the Algorithm Recognize-HH-free. In turn, if we assume that we have an adjacency-list representation of the input graph $G$ and that we use two auxiliary arrays (of size $n$ ) to mark the neighbors of $v$ and of $y$, it is not difficult to see that all three steps of the certificate computation take $O(n+m)$ time and $O(n)$ space, where $n, m$ are the numbers of vertices and edges of $G$, respectively. Therefore, we have:

Theorem 3.3 The Algorithm Recognize-HH-free can be easily augmented to provide a certificate whenever it decides that the input graph $G$ is not $H H$-free; if $G$ has $n$ vertices and $m$ edges, the certificate computation takes $O(n+m)$ time and $O(n)$ space.

\section{Recognition of HHD-free and WPO-Graphs}

In this section, we present two applications of the ideas and of the algorithm for recognizing HH-free graphs. In particular, we show how to use the $\mathrm{HH}$-free graph recognition algorithm in order to recognize HHD-free graphs within the same time and space complexity, and how the ideas can be used to determine if the complement of a given graph is HH-free so that we can recognize WPO-graphs.

(ii) It must be noted that vertex $x$ may have been added in $A(u)$ more than once by different vertices; yet, $x$ and any of these vertices suffice for our purposes. 
Algorithm Recognize-HHD-free

1. if the input graph $G$ is not $\mathrm{HH}$-free

then return("the graph is not HHD-free");

2. Run LexBFS on $G$ starting at an arbitrary vertex $w$, and let $\left(v_{1}, v_{2}, \ldots, v_{n}\right)$ be the resulting ordering, where $v_{n}=w$.

3. for $i=1,2, \ldots, n-5$ do

if $v_{i}$ is not semi-simplicial in $G\left[\left\{v_{i}, v_{i+1}, \ldots, v_{n}\right\}\right]$

then return("the graph is not HHD-free");

4. return("the graph is HHD-free").

Fig. 7: The algorithm for determining whether a graph $G$ is HHD-free.

\subsection{HHD-free Graphs}

Our HHD-free graph recognition algorithm is motivated by the corresponding algorithm of Hoàng and Sritharan [9], which in turn is motivated by the work of Hoàng and Khouzam [8] and relies on the following characterization of HHD-free graphs proved by Jamison and Olariu:

Theorem 4.1 (Jamison and Olariu [10]) The following two statements are equivalent:

(i) The graph $G$ is HHD-free;

(ii) For every induced subgraph $H$ of the graph $G$, every ordering of vertices of $H$ produced by LexBFS is a semi-perfect elimination.

In fact, we could use the Algorithm Not-in- $\operatorname{HHB}(G, v)$ in Hoàng and Sritharan's HHD-free graph recognition algorithm in order to determine if vertex $v$ is high, and we would achieve the improved time and space complexities stated in this paper. However, we can get the much simpler algorithm which we give in Figure 7

Note that, after step 1 , we need only check whether the input graph $G$ contains a domino; this is why, we only process the $n-5$ vertices $v_{1}, v_{2}, \ldots, v_{n-5}$ in step 3 Additionally, it is important to observe that, for all $i=1,2, \ldots, n$, the ordering $\left(v_{i}, v_{i+1}, \ldots, v_{n}\right)$ is an ordering which can be produced by running LexBFS on the subgraph $G\left[\left\{v_{i}, v_{i+1}, \ldots, v_{n}\right\}\right]$ starting at vertex $v_{n}$. The correctness of the algorithm follows from Theorem 4.1 and the fact that if the currently processed vertex $v_{i}$ in step 3 is semi-simplicial then clearly it cannot participate in a domino (note that none of the vertices of a domino is semi-simplicial in any graph containing the domino as induced subgraph).

Time and Space Complexity. Let $n$ and $m$ be the number of vertices and edges of the input graph $G$. According to Corollary 3.1, step 1 takes $O(n \min \{m \alpha(n, n), m+n \log n\})$ time and $O(n+m)$ space. step 2 takes $O(n+m)$ time and space [5; 16]. The construction of the subgraphs $G\left[\left\{v_{i}, v_{i+1}, \ldots, v_{n}\right\}\right]$ in step 3 can be done in a systematic fashion by observing that $G\left[\left\{v_{1}, v_{2}, \ldots, v_{n}\right\}\right]=G$ and that $G\left[\left\{v_{i+1}, v_{i+2}, \ldots, v_{n}\right\}\right]$ can be obtained from $G\left[\left\{v_{i}, v_{i+1}, \ldots, v_{n}\right\}\right]$ by removing vertex $v_{i}$ and all its incident edges; if the graph $G$ is stored using a (doubly-connected) adjacency-list representation with 
pointers for every edge $a b$ connecting the record storing $b$ in the adjacency list of $a$ to the record storing $a$ in the adjacency list of $b$ and back, then obtaining $G\left[\left\{v_{i+1}, v_{i+2}, \ldots, v_{n}\right\}\right]$ from $G\left[\left\{v_{i}, v_{i+1}, \ldots, v_{n}\right\}\right]$ takes time proportional to the degree of $v_{i}$ in $G\left[\left\{v_{i}, v_{i+1}, \ldots, v_{n}\right\}\right]$ and hence $O\left(\operatorname{deg}\left(v_{i}\right)\right)$ time, where $\operatorname{deg}\left(v_{i}\right)$ denotes the degree of vertex $v_{i}$ in $G$. Additionally, in order to check whether a vertex is semisimplicial, we take advantage of the following result of Hoàng and Khouzam (which was also used in [9]):

Theorem 4.2 (Hoàng and Khouzam [8]) Let $G$ be a graph and $x$ be a semi-simplicial vertex of $G$. If $x$ is not simplicial, then each big co-component of the subgraph $G[N(x)]$ is a module of $G$.

(A connected component or co-component of a graph is called big if it has at least two vertices; we also note that if a vertex $x$ is simplicial then none of the co-components of the subgraph $G[N(x)]$ is big.) Since computing the subgraph induced by the neighbors of vertex $v_{i}$ in $G\left[\left\{v_{i}, v_{i+1}, \ldots, v_{n}\right\}\right]$, computing its cocomponents, and testing whether a vertex set is a module in $G\left[\left\{v_{i}, v_{i+1}, \ldots, v_{n}\right\}\right]$ can all be done in time and space linear in the size of $G\left[\left\{v_{i}, v_{i+1}, \ldots, v_{n}\right\}\right]$, step 3 takes a total of $O\left(\sum_{i}\left(n+m+\operatorname{deg}\left(v_{i}\right)\right)\right)=$ $O(n m)$ time and $O(n+m)$ space. Finally, step 4 takes constant time. Therefore, we obtain the following theorem.

Theorem 4.3 Let $G$ be an undirected graph on $n$ vertices and $m$ edges. Then, Algorithm Recognize$H H D$-free determines whether $G$ is an HHD-free graph in $O(n \min \{m \alpha(n, n), m+n \log n\})$ time and $O(n+m)$ space.

\subsection{Welsh-Powell Opposition Graphs}

Our algorithm for recognizing WPO-graphs relies on the fact that a graph $G$ is a WPO-graph if and only if $G$ is HHP-free and its complement $\bar{G}$ is HH-free, which follows from the following characterization due to Olariu and Randall [15]:

Theorem 4.4 (Olariu and Randall [15]) A graph $G$ is a WPO-graph if and only if G contains no induced $C_{5}, P_{5}$, house, or " $P$ ".

Eschen et al. [4] described an $O(n \mathrm{~m})$-time algorithm for recognizing whether a graph $G$ on $n$ vertices and $m$ edges is HHP-free by using the modular decomposition tree of $G$ and Theorem 4.2 due to Hoàng and Khouzam [8]. We next show that we can detect whether the complement $\bar{G}$ of $G$ contains a hole or a house in $O(n m)$ time. Combining these two algorithms, we get an $O(n m)$-time algorithm for recognizing WPO-graphs.

The obvious approach of running Algorithm Recognize-HH-free on the complement $\bar{G}$ of $G$, in order to check whether $\bar{G}$ contains a hole or a house, proves to be expensive. Thus, instead, we process the vertices of $G$ in turn; for each vertex $v$, we consider a (different) auxiliary graph $\widehat{G}_{v}$ as follows:

$$
\begin{aligned}
\circ V\left(\widehat{G}_{v}\right)= & V(G) \\
\circ E\left(\widehat{G}_{v}\right)= & \{v y \mid y \in M(v)\} \\
& \cup\{x y \mid x \in N(v), y \in M(v), \text { and } x y \notin E(G)\} \\
& \cup\left\{x x^{\prime} \mid x, x^{\prime} \in N(v) \text { and } x x^{\prime} \notin E(G)\right\}
\end{aligned}
$$


Note that in $\bar{G}$ the neighbors of $v$ are the non-neighbors $M(v)$ of $v$ in $G$, and its non-neighbors are the neighbors $N(v)$ of $v$ in $G$. Thus, the graph $\widehat{G}_{v}$ is precisely $\bar{G}$ without any edges between neighbors of $v$. This implies that:

(i) $v$ cannot be the top of a house or a building in $\widehat{G}_{v}$, and

(ii) if $v$ is the top of a house or a building in $\bar{G}$, then it belongs to a hole in $\widehat{G}_{v}$.

Then, in order to check whether vertex $v$ belongs to a hole or is the top of a house or a building in $\bar{G}$, it suffices to execute Algorithm Not-in- $\operatorname{HHB}\left(\widehat{G}_{v}, v\right)$, and answer "true" if and only if Algorithm Not-in$\operatorname{HHB}\left(\widehat{G}_{v}, v\right)$ returns "false." Thus, we have the following result.

Lemma 4.1 The vertex $v$ belongs to a hole or is the top of a house or a building in $\bar{G}$ if and only if Algorithm Not-in-HHB( $\left.\widehat{G}_{v}, v\right)$ returns "false."

Lemma 4.1 and the complexity of Algorithm Not-in-HHB implies the following theorem.

Theorem 4.5 Let $G$ be an undirected graph on $n$ vertices and $m$ edges. Then, the algorithm that we described in this section determines whether the complement $\bar{G}$ is an HH-free graph in $O(n m)$ time and $O\left(n^{2}\right)$ space.

Proof: It suffices to establish the theorem for a connected graph $G$. If $G$ is not connected, then, because the complement of a house and the complement of a hole are both connected, we work with the connected components of $G$, which can be computed in $O(n+m)$ time and space [3]. Thus, suppose that $G$ is connected; then, $n=O(m)$. In order to facilitate the construction of the auxiliary graphs, we compute and store the adjacency matrix of $G$, which takes $O\left(n^{2}\right)$ time and space. Then, for any vertex $v$ of $G$, the graph $\widehat{G}_{v}$ has $n$ vertices and $O\left(n+n \operatorname{deg}(v)+\operatorname{deg}^{2}(v)\right)=O(n \operatorname{deg}(v))$ edges, where $\operatorname{deg}(v)$ is the degree of $v$ in $G$. We can easily obtain an adjacency-list representation of $\widehat{G}_{v}$ by computing the sets $N(v)$ of neighbors and $M(v)$ of non-neighbors of $v$ in $G$, and by taking advantage of the adjacency matrix of $G$; the computation of the sets $N(v)$ and $M(v)$ takes $O(n+m)$ time and $O(n)$ space, while the construction of the adjacency lists of $\widehat{G}_{v}$ is completed in $O(m+n \operatorname{deg}(v))$ additional time and $O(n \operatorname{deg}(v))$ space for a total of $O(m+n \operatorname{deg}(v))$ time and $O(n \operatorname{deg}(v))$ space. The execution of Algorithm Not-in-HHB $\left(\widehat{G}_{v}, v\right)$ takes $O(\min \{(n+n \operatorname{deg}(v)) \alpha(n, n), n \operatorname{deg}(v)+\operatorname{deg}(v) \log \operatorname{deg}(v)\})=$ $O(n \operatorname{deg}(v)+\operatorname{deg}(v) \log \operatorname{deg}(v))=O(n \operatorname{deg}(v))$ time (Theorem 3.2, note that $k=\operatorname{deg}(v))$. Thus, we can determine whether the vertex $v$ belongs to a hole in $\widehat{G}_{v}$ in $O(m+n \operatorname{deg}(v))$ time and $O(n \operatorname{deg}(v))$ space. Then, the total time required, i.e., the time required for the computation of the adjacency matrix of $G$ and the processing of all the vertices, is

$$
O\left(n^{2}+\sum_{v}(m+n \operatorname{deg}(v))\right)=O\left(n m+n \sum_{v} \operatorname{deg}(v)\right)=O(n m) .
$$

The space complexity is $O\left(n^{2}\right)$ since the space taken by each auxiliary graph $\widehat{G}_{v}$ can be reused after the processing of $v$.

From Theorem 4.5 and the result of Eschen et al. [4] (i.e., HHP-free graphs can be recognized in $O(n \mathrm{~m})$ time and $O(n+m)$ space), we obtain the following theorem.

Theorem 4.6 Let $G$ be an undirected graph on $n$ vertices and $m$ edges. Then, it can be determined whether $G$ is a WPO-graph in $O(n m)$ time and $O\left(n^{2}\right)$ space. 


\section{Concluding Remarks}

We have presented recognition algorithms for the classes of $\mathrm{HH}$-free and HHD-free graphs running in $O(n \min \{m \alpha(n, n), m+n \log n\})$ time, and for WPO-graphs running in $O(n m)$ time, where $n$ is the number of vertices and $m$ is the number of edges of the input graph. Our proposed algorithms are simple and require $O(n+m)$ and $O\left(n^{2}\right)$ space, respectively. Moreover, our HH-free graph recognition algorithm can be easily augmented to yield a certificate (a hole or a house) whenever it decides that the input graph is not HH-free.

We leave as an open problem the designing of $O(n \mathrm{~m})$-time algorithms for recognizing $\mathrm{HH}$-free and HHD-free graphs; note that an $O(n m)$-time algorithm for recognizing HH-free graphs directly implies an $O(n m)$-time recognition algorithm for HHD-free graphs. Additionally, in light of the $O(n m)$-time recognition of $P_{4}$-comparability, $P_{4}$-simplicial, bipolarizable, and WPO-graphs, it would be worth investigating whether the recognition of brittle and semi-simplicial graphs is inherently more difficult.

\section{References}

[1] A. Brandstädt, V.B. Le, and J.P. Spinrad, Graph classes: A survey, SIAM Monographs on Discrete Mathematics and Applications, 1999.

[2] V. Chvátal, Perfectly ordered graphs, Annals of Discrete Math. 21, 63-65, 1984.

[3] T.H. Cormen, C.E. Leiserson, R.L. Rivest, and C. Stein, Introduction to Algorithms (2nd edition), MIT Press, Inc., 2001.

[4] E.M. Eschen, J.L. Johnson, J.P. Spinrad, and R. Sritharan, Recognition of some perfectly orderable graph classes, Discrete Appl. Math. 128, 355-373, 2003.

[5] M.C. Golumbic, Algorithmic Graph Theory and Perfect Graphs, Academic Press, Inc., 1980.

[6] R. Hayward, Meyniel weakly triangulated graphs I: co-perfect orderability, Discrete Appl. Math. 73, 199-210, 1997.

[7] C.T. Hoàng, On the complexity of recognizing a class of perfectly orderable graphs, Discrete Appl. Math. 66, 219-226, 1996.

[8] C.T. Hoàng and N. Khouzam, On brittle graphs, J. Graph Theory 12, 391-404, 1988.

[9] C.T. Hoàng and R. Sritharan, Finding houses and holes in graphs, Theoret. Comput. Sci. 259, 233-244, 2001.

[10] B. Jamison and S. Olariu, On the semi-perfect elimination, Adv. Appl. Math. 9, 364-376, 1988.

[11] R.M. McConnell and J. Spinrad, Linear-time modular decomposition and efficient transitive orientation, Proc. 5th Annual ACM-SIAM Symp. on Discrete Algorithms (SODA'94), 536-545, 1994.

[12] M. Middendorf and F. Pfeiffer, On the complexity of recognizing perfectly orderable graphs, Discrete Math. 80, 327-333, 1990.

[13] S. Olariu, All variations on perfectly orderable graphs, J. Combin. Theory Ser. B 45, 150-159, 1988.

[14] S. Olariu, Weak bipolarizable graphs, Discrete Math. 74, 159-171, 1989.

[15] S. Olariu and J. Randall, Welsh-Powell opposition graphs, Inform. Process. Lett. 31, 43-46, 1989.

[16] D.J. Rose, R.E. Tarjan, and G.S. Lueker, Algorithmic aspects of vertex elimination on graphs, SIAM J. Comput. 5, 266-283, 1976.

[17] R.E. Tarjan, Data structures and network algorithms, Society for Industrial and Applied Mathematics, 1983.

[18] D.J.A. Welsh and M.B. Powell, An upper bound on the chromatic number of a graph and its applications to timetabling problems, Comput. J. 10, 85-87, 1967. 